\title{
Giant Vibrations of Impurity Atoms on a Crystal Surface
}

\section{Citation}

Martinez, Robert E., E. Fontes, J. A. Golovchenko, and J. R. Patel. 1992. Giant Vibrations of Impurity Atoms on a Crystal Surface. Physical Review Letters 69, no. 7: 1061-1064. doi:10.1103/ physrevlett.69.1061.

\section{Published Version}

doi:10.1103/PhysRevLett.69.1061

\section{Permanent link}

http://nrs.harvard.edu/urn-3:HUL.InstRepos:29407030

\section{Terms of Use}

This article was downloaded from Harvard University's DASH repository, and is made available under the terms and conditions applicable to Other Posted Material, as set forth at http:// nrs.harvard.edu/urn-3:HUL.InstRepos:dash.current.terms-of-use\#LAA

\section{Share Your Story}

The Harvard community has made this article openly available.

Please share how this access benefits you. Submit a story.

Accessibility 


\title{
Giant Vibrations of Impurity Atoms on a Crystal Surface
}

\author{
R. E. Martinez, (1) E. Fontes, ${ }^{(2),(a)}$ J. A. Golovchenko, ${ }^{(1),(3)}$ and J. R. Patel $^{(2)}$ \\ ${ }^{(1)}$ Physics Department, Harvard University, Cambridge, Massachusetts 02138 \\ ${ }^{(2)}$ AT\& T Bell Laboratories, Murray Hill, New Jersey 07974 \\ ${ }^{(3)}$ Rowland Institute for Science, Cambridge, Massachusetts 02142
}

(Received 7 October 1991)

\begin{abstract}
We have measured extremely large anisotropic thermal vibrations of gallium adatoms on a $\mathrm{Si}(111)$ surface using $x$-ray standing waves. At $830 \mathrm{~K}$ the rms amplitude normal to the surface is $0.47 \pm 0.02 \AA$ while the parallel amplitude is $0.30 \pm 0.02 \AA$. The temperature dependence of these amplitudes deviates significantly from that expected from Debye theory and suggests a significant thermal softening of the surface bonding at elevated temperatures.
\end{abstract}

PACS numbers: $61.10 . \mathrm{Lx}, 63.20 .-\mathrm{e}, 63.70 .+\mathrm{h}, 68.35 . \mathrm{Ja}$

The past several decades have witnessed a remarkable development of techniques necessary to study crystal surfaces. This has resulted in new and dramatic insights into surface structural and electronic phenomena. Our own interests have centered on semiconductors, in part because of the importance of these materials for electronic devices and in part because of the rich phenomena their covalently bonded surfaces support. Experimental observations and heuristic explanations have often been closely followed by more detailed theoretical understanding based on quantum-mechanical models. Of particular importance have been the results of local-density-functional calculations [1] in predicting the energies and surface stresses of different surface reconstructions on both clean and chemisorbed surfaces.

The high-temperature behavior of semiconductor surfaces has received increasing experimental attention in recent years because of the importance of understanding phenomena like surface phase transitions, diffusion [2], and epitaxial growth. It seems likely that theoretical insight will flow from large-scale molecular dynamics calculations [3]. Here the thermally stimulated classical motions of large arrays of atoms are calculated using many-body potentials gleaned either from experiments or further quantum-mechanical calculations.

In order to provide experimental insight into the thermal behavior of surface atoms we decided to use the $x$-ray standing-wave method to determine the absolute vibrational amplitudes of impurity atoms on a silicon surface as a function of temperature. The study of surface impurity-atom vibration is important in itself and easier than that of host surface atoms because signals from the latter are notoriously difficult to isolate experimentally from those of subsurface host atoms. Scattering probes like electrons [4], x rays [5], and helium atoms [6] can provide information here but they either average over several surface layers or do not distinguish the atoms being measured. Surface extended x-ray-absorption spectroscopy, on the other hand, can distinguish the atom being measured if it is an impurity, but only the relative amplitude of atomic vibration can be determined [7]. Fi- nally ion channeling and blocking techniques [8] can be used to determine impurity vibrations in favorable cases although this technique is not appropriate for large vibrational amplitudes and incommensurate surfaces.

Our program to measure surface vibrations using $x$-ray standing waves began with the study of monolayer coverages of surface substitutional arsenic atoms on Si(111). This is an extremely well understood surface at low temperatures where theory and experiment agree on the atomic location to a hundredth of an angstrom $[9,10]$. Because of space limitations here, these high-temperature results will be published elsewhere. Let it suffice to say that there were no major surprises and good agreement with molecular dynamics calculations has been obtained with a minimum of modeling using three-body potentials. On the other hand, the case we studied next, and on which we report in detail below, was gallium on Si(111). Here the atoms occupy a nonsubstitutional surface site [11] and exhibit surprisingly large vibrational amplitudes and non-Debye behavior that is as yet not understood.

We begin with a brief discussion of the $x$-ray standing-wave method which was first applied to surface problems by Cowan, Golovchenko, and Robbins [12]. In this method the interference between a monochromatic incident $x$-ray beam and the Bragg reflected beam from a perfect crystal form a standing $x$-ray field near the surface that is commensurate with, and has the periodicity of, the bulk crystal charge-density Fourier component responsible for the reflection. The standing-wave phase fronts can be moved continuously relative to this charge density by tuning the angle of incidence $\Theta$ through the finite angular width of the Bragg reflection. The structure of an impurity layer localized at the surface can be determined by monitoring the characteristic fluorescence radiation from these atoms excited by the x-ray standing waves. The fluorescence yield from an impurity layer, with (normalized) density

$$
\rho(r)=(1 / N) \sum_{j} \delta\left(r-r_{j}-u_{j}\right),
$$

where $u_{j}$ is the instantaneous thermal displacement of the 
$j$ th impurity atom, is given by

$$
Y(\Theta)=1+R(\Theta)+2|R(\Theta)|^{1 / 2} F_{G} \cos \left[2 \pi P_{G}-\phi(\Theta)\right] .
$$

$R(\Theta)$ is the Darwin-Prins reflectivity of the crystal, and $\phi(\Theta)$ is the phase of the standing $x$-ray wave field relative to the charge-density component of wave number $G$ $=2 \pi / d$, with $d$ the periodic diffraction interplanar spacing. The fluorescence yield is normalized to unity far from the Bragg condition where $R=0$. In the above expression [13]

$$
F_{G} \exp \left[2 \pi i P_{G}\right]=(1 / N) \exp (-M) \sum_{j} \exp \left(i G \cdot r_{j}\right)
$$

and $M$ is the Debye-Waller exponent,

$$
M=\left\langle(G \cdot u)^{2}\right\rangle / 2+O\left(\left\langle(G \cdot u)^{4}\right\rangle\right) .
$$

$P_{G}$ measures the (dimensionless) position of the impurity layer relative to the bulk extrapolated diffraction planes, in units of the bulk interplanar spacing. $F_{G}$, the "coherent fraction," measures how sharply distributed around $P_{G}$ the atoms are positioned. A low-temperature coherent fraction less than unity suggests a broadened static distribution of overlayer atoms. More importantly because $F_{G}$ is proportional to the Debye-Waller term $\exp (-M)$, a systematic study of the temperature dependence of the coherent fraction allows the amplitude of surface vibrations to be measured accurately.

As mentioned earlier, gallium on $\mathrm{Si}(111)$ was chosen as a nontrivial case to study. At coverages $\approx \frac{1}{3}$ monolayer (ML) gallium atoms are not substitutional like arsenic but are known to occupy $T 4$ sites in a $(\sqrt{3}$ $\times \sqrt{3}) R 30$ reconstruction on this surface. This structure with $\frac{1}{3}$ monolayer coverage has all dangling bonds saturated. No other reconstruction is known on this surface. The low-energy electron-diffraction (LEED) pattern remains $(\sqrt{3} \times \sqrt{3}) R 30$ up to a temperature of $850 \mathrm{~K}$ at which point gallium begins to desorb and the surface reverts to a $7 \times 7$ reconstruction characteristic of clean $\mathrm{Si}(111)$. It follows that the surface neither melts nor undergoes any structural static displacement or phase transitions prior to gallium desorption. Recent calculations [14] and experiments [15] show that this surface is under tension which argues strongly against any long-wavelength static buckling which might elude LEED observations. Finally, roughening transitions, which are sometimes observed on metal surfaces, are experimentally unknown, undetected, and unexpected on this semiconductor surface. The experiment was therefore optimized for measuring thermal vibrations which are the only type of surface excitations to be expected.

$\mathrm{X}$-ray standing-wave measurements were performed at the AT\&T Bell Laboratories beam line X-15A at the BNL National Synchrotron Light Source facility using 12.6-keV x rays. Silicon samples were oriented and polished parallel to the (111) crystal planes to an accuracy better than $0.1 \mathrm{deg}$, and then chemically cleaned using the Shiraki procedure [16] before introduction into the experimental UHV system (base pressure $1 \times 10^{-10}$ torr). Sample temperatures were monitored by an infrared optical pyrometer and a Chromel-Alumel thermocouple was mounted beneath the sample. Comparisons with samples having embedded thermocouples showed that all temperatures quoted here are accurate to $\pm 10 \mathrm{~K}$.

After cleaning in the UHV chamber by heating to $1160 \mathrm{~K}$ for $2 \mathrm{~min}$, the sample exhibited a sharp Si(111) $7 \times 7$ LEED pattern. Auger electron spectroscopy and $x$-ray fluorescence measurements showed it to be free of detectable contaminates. Gallium was deposited by heating the sample to $945 \mathrm{~K}$ and exposing it to a previously outgassed gallium effussion cell operating at 1100 $\mathrm{K}$. When the sample cooled, a sharp uniform LEED pattern was obtained, containing a mixture of $(\sqrt{3} \times \sqrt{3}) R 30$ and $7 \times 7$ diffraction spots. Gallium coverage was determined to be $0.1 \pm 0.01 \mathrm{ML}$ by measuring the ratio of $\mathrm{Ga}$ $K \alpha$ to $\mathrm{Si} K \alpha$ x-ray fluorescence yields far away from the Bragg condition, and comparing this ratio to that of a calibrated gallium-ion-implanted sample.

The position and structural perfection of the gallium layer were then measured using $\mathrm{x}$-ray standing waves. The sample was annealed at $840 \mathrm{~K}$ until the gallium overlayer developed an extremely high room-temperature coherent fraction $F_{111}$ (normal to the surface) of 0.98 \pm 0.01 . The position of the layer, $P_{111}$, was $0.58 \pm 0.01$ which corresponds to a mean gallium atom height of $1.46 \pm 0.03 \AA$ above the upper half of the bulk extrapolated $\mathrm{Si}(111)$ double layer, in excellent agreement with previous standing-wave results [11]. These same measurements were carried out for the $(11 \overline{1})$ plane which is inclined at $70.53 \mathrm{deg}$ to the $(11 \overline{1})$ surface. $F_{11 \overline{1}}$ and $P_{11 \overline{1}}$ were found to be $0.99 \pm 0.01$ and $0.34 \pm 0.01$, respectively. The standing-wave raw data, fits, and atomic position are shown in Fig. 1. The measurement using both atomic planes allows a unique quantitative assignment of the gallium atom site which confirms the scanning-tunnelingmicroscope $T 4$ site observation [11].

As the sample was heated, the coherent fraction for gallium atoms in both the [111] and [11i] directions decreased as can be seen in Fig. 1. We take this to be due to thermal vibrations and deduce the mean-square amplitude along these directions from

$$
\left\langle u^{2}\right\rangle=-2 \ln [F(T)] /|G|^{2} .
$$

Figure 2 shows plots of the temperature dependence of $\left\langle u^{2}\right\rangle$ for gallium atoms in the $(\sqrt{3} \times \sqrt{3}) R 30$ reconstruction on the Si(111) surface.

The most striking feature of the data is the extremely large $0.47 \pm 0.02 \AA$ vibration amplitude normal to the surface obtained at $830 \mathrm{~K}$. Equally important is the strong deviation from a linear temperature dependence predicted by a Debye model. It is remarkable that in spite of these very large vibrational amplitudes there is no 


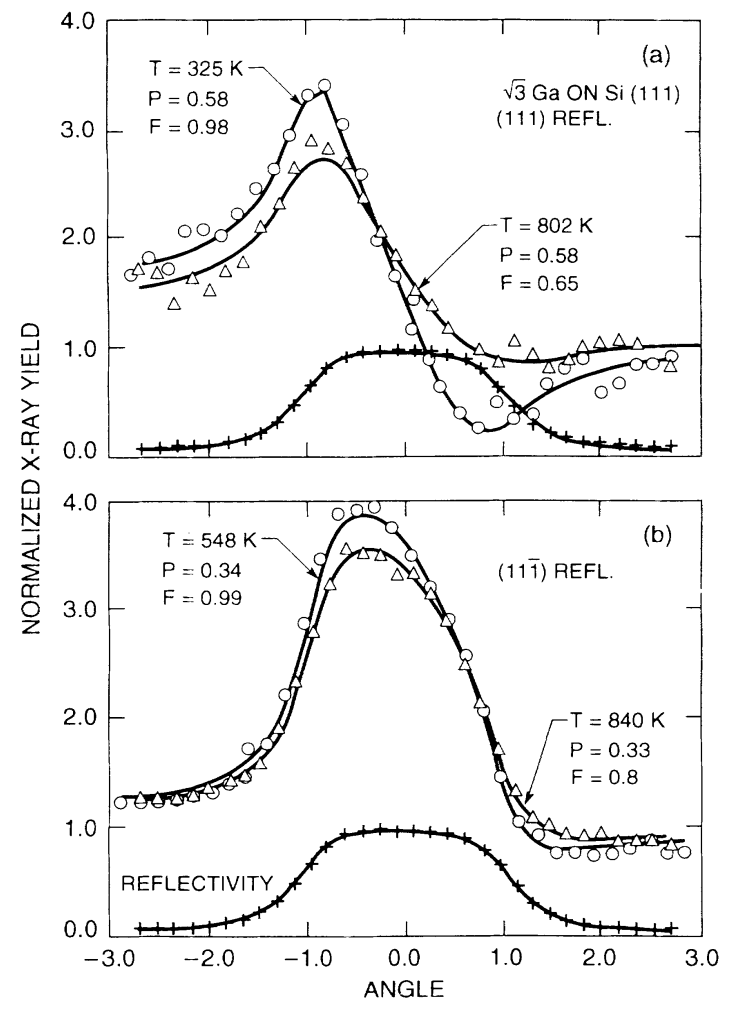

FIG. 1. Reflectivity and fluorescence yield for $\mathrm{Ga}$ on $\operatorname{Si}(111)(\sqrt{3} \times \sqrt{3}) R 30$. (a) (111) reflection. (b) (11i) reflection.

shift, to within our $0.03 \AA$ accuracy, in the mean gallium atom position at elevated temperatures.

By comparison the vibrational amplitude along [11i $]$ is seen to reach a maximum of $0.33 \pm 0.02 \AA$ at $830 \mathrm{~K}$, which implies, assuming surface perpendicular and parallel vibrational amplitudes are independent of one another, a parallel vibrational amplitude of $0.30 \pm 0.02 \AA$. Again no shift in mean atom position is observed to the highest temperature of the measurement.

The large magnitude of the above vibrational amplitudes can perhaps be appreciated best by comparison with that for the incommensurate $6.3 \times 6.3$ reconstruction obtained by increasing the gallium coverage to about 1 ML. Gallium atoms in this layer are believed to bond with and become nearly coplanar with a single layer of $\mathrm{Si}(111)$ atoms [17]. Only the perpendicular component can be determined from $\mathrm{x}$-ray standing waves in this incommensurate surface; the results are shown in Fig. 2. (Incidentally, perpendicular amplitudes for surface substitutional arsenic atoms are very similar to the $\mathrm{Ga}$ $6.3 \times 6.3$ data.) Note that only at the highest temperatures $(\approx 800 \mathrm{~K})$ studied does a strong deviation from Debye-like behavior seem to begin.

Returning to the $(\sqrt{3} \times \sqrt{3}) R 30$ data we can draw a number of preliminary conclusions. It is not surprising that there is no shift in the mean position parallel to the

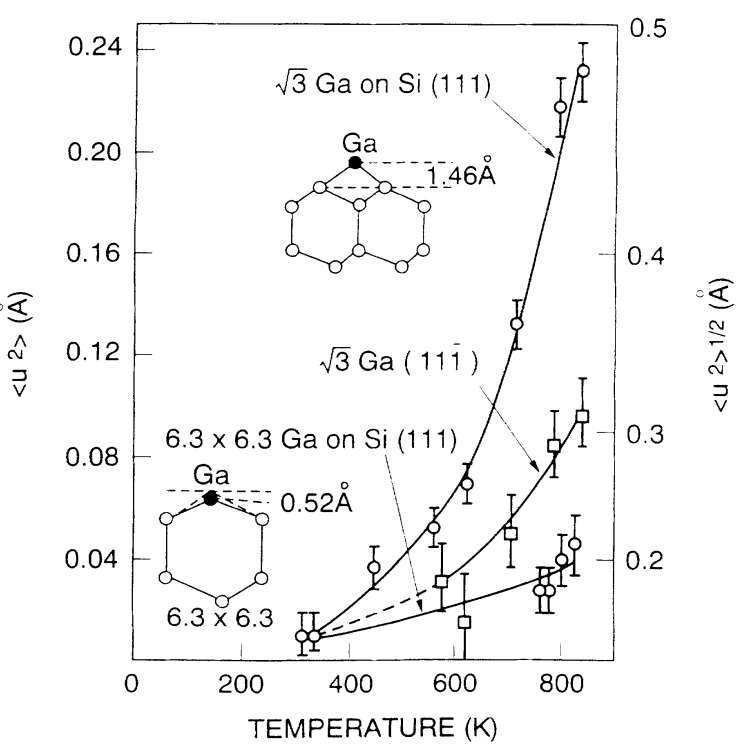

FIG. 2. Temperature dependence of the mean-square amplitude of $\mathrm{Ga}$ atom vibrations on the $\operatorname{Si}(111)(\sqrt{3} \times \sqrt{3}) R 30$ and $6.3 \times 6.3$ surfaces. Curves are drawn to guide the eye. The inset shows the Ga atom location on the $(\sqrt{3} \times \sqrt{3}) R 30$ and $6.3 \times 6.3$ reconstructions.

surface at elevated temperature, due to the high symmetry of the $T 4$ binding site in this plane. It is surprising, however, that there is no shift perpendicular to the surface. This suggests that cubic terms in the bindingpotential anharmonicity normal to the surface must be quite small. A possible explanation may involve significant lateral relaxation of the neighboring silicon atoms as the gallium atom moves towards the surface. Deviations from Debye-like behavior show that negative fourth-order terms in the potential are already important when the normal vibrational amplitudes exceed $0.1 \AA$ just above room temperature. This we also find quite surprising.

Doak [6] has measured the spectrum of phonons on the gallium $(\sqrt{3} \times \sqrt{3}) R 30$ surface using low-energy atomic helium atom scattering. Surface phonon dispersion curves measured along the $[11 \overline{2}]$ azimuth display a flat optic mode at approximately $11.5 \mathrm{meV}$, and an acoustic mode which bends and flattens abruptly near the Brillouin-zone center, reaching a plateau of approximately $10 \mathrm{meV}$ at the zone boundary. Assuming that the weight of these low-lying vibrational eigenstates is concentrated primarily on the gallium atoms, their flat dispersion is likely the result of (anisotropic) Einstein oscillator motion of nearly independent gallium adatoms [6]. The large separation of gallium adatoms $(6.65 \AA)$ tends to support this argument. With this assumption the resonant frequency $\Omega$ of the vibrations normal to the surface is

$$
\Omega=\left[k_{b} T / M\left\langle u_{\perp}^{2}\right\rangle\right]^{1 / 2},
$$




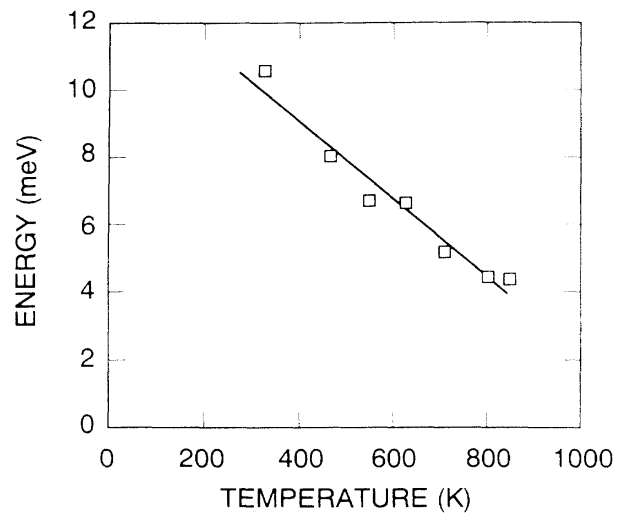

FIG. 3. Temperature dependence of the resonant frequency of $\mathrm{Ga}$ atom vibrations normal to the $\mathrm{Si}(111)(\sqrt{3} \times \sqrt{3}) R 30$ surface, calculated using Eq. (6).

where $M$ is the mass of gallium. Using our roomtemperature value of $\left\langle u_{\perp}^{2}\right\rangle$ we obtain good agreement with the zone-boundary frequency discussed in Ref. [6]. If one chooses to model the extra large vibration amplitudes obtained at larger temperatures as resulting from a "softening" of a harmonic gallium binding potential one obtains the results plotted in Fig. 3. The softening is dramatically shown as a reduction in the normal resonant mode frequency by nearly a factor of 4 at $830 \mathrm{~K}$. We believe either large shifts or broadening of the helium inelastic scattering line (relative to its room-temperature values) will be observed when this system is studied at elevated temperatures.

In conclusion, we have measured extremely large vibrational amplitudes of gallium atoms on the $\mathrm{Si}(111)(\sqrt{3}$ $\times \sqrt{3}) R 30$ surface using $x$-ray standing waves. Our experiments clearly demonstrate the power of this technique in directly measuring thermal vibration amplitudes. More importantly, we have discovered that this seemingly simple surface reconstruction displays remarkable and unexpected vibrational phenomena, which challenge our understanding of surface vibrational dynamics. We hope our results will inspire further theoretical and experimental studies of this system.

We are indebted to Paul Freeland for his masterful preparations of silicon samples, and to George Gilmer, Bruce Doak, and Ed Chaban for extremely helpful discussions. This work was supported in part by the U.S. Department of Energy, under Contract No. DE-FG02-
89ER-45399, and by the Harvard Materials Research Laboratory under NSF Grant No. DMR-89-20490.

(a) Current address: Cornell High Energy Synchrotron Source, Ithaca, NY 14853.

[1] I. Stich, M. C. Payne, R. D. King-Smith, J-S. Lin, and L. J. Clarke, Phys. Rev. Lett. 68, 1351 (1992); K. D. Brummer, M. Needles, B. E. Larson, and J. D. Joannopoulos, Phys. Rev. Lett. 68, 1355 (1992); see also Ref. [14] below.

[2] E. Ganz, S. K. Theiss, I.-S. Hwang, and J. Golovchenko, Phys. Rev. Lett. 68, 1567 (1992); G. A. Reider, U. Höfer, and T. F. Heinz, Phys. Rev. Lett. 66, 1994 (1991); Y. W. Mo, J. Kleiner, M. B. Webb, and M. G. Lagally, Phys. Rev. Lett. 66, 1998 (1991).

[3] C. Roland and G. H. Gilmer, Phys. Rev. Lett. 67, 3188 (1991).

[4] E. G. McRae and R. A. Malic, Phys. Rev. Lett. 58, 1437 (1987).

[5] S. G. J. Mochrie, D. M. Zehner, B. M. Ocko, and D. Gibbs, Phys. Rev. Lett. 64, 2925 (1990).

[6] R. B. Doak, J. Vac. Sci. Technol. B 7, 1252 (1989).

[7] F. Sette, T. Hashizume, R. F. Comin, A. A. MacDowell, and P. H. Citrin, Phys. Rev. Lett. 61, 1384 (1988).

[8] J. F. van der Veen and J. W. M. Frenken, Surf. Sci. 251/252, 1 (1991), and references therein.

[9] J. R. Patel, J. A. Golovchenko, P. E. Freeland, and H.-J. Gossmann, Phys. Rev. B 36, 7715 (1987).

[10] R. I. G. Uhrberg, R. D. Bringans, M. A. Olmstead, R. Z. Bachrach, and J. E. Northrup, Phys. Rev. B 35, 3945 (1987).

[11] J. Zegenhagen, J. R. Patel, P. E. Freeland, D. M. Chen, J. A. Golovchenko, P. Bedrossian, K. Mortensen, and J. E. Northrup, Phys. Rev. B 39, 1298 (1989).

[12] P. L. Cowan, J. A. Golovchenko, and M. F. Robbins, Phys. Rev. Lett. 44, 1680 (1980).

[13] N. Hertel, G. Materlik, and J. Zegenhagen, Z. Phys. B 58, 199 (1985).

[14] R. D. Meade and D. Vanderbilt, Phys. Rev. Lett. 63, 1404 (1989).

[15] R. E. Martinez, W. M. Augustyniak, and J. A. Golovchenko, Phys. Rev. Lett. 64, 1035 (1990).

[16] A. Ishizaka, N. Nakagawa, and Y. Shiraki, in Proceedings of the Second International Conference on Molecular Beam Epitaxy and Related Clean Surface Techniques (Japan Society of Applied Physics, Tokyo, 1982), p. 183.

[17] D. M. Chen, J. A. Golovchenko, P. Bedrossian, and K. Mortensen, Phys. Rev. Lett. 61, 2867 (1988) 